Portland State University

PDXScholar

$10-2020$

\title{
Genetically Encoded Phase Contrast Agents for Digital Holographic Microscopy
}

\author{
Arash Farhadi \\ California Institute of Technology \\ Manuel Bedrossian \\ California Institute of Technology \\ Justin Lee \\ California Institute of Technology \\ Gabrielle H. Ho \\ California Institute of Technology \\ Mikhail G. Shapiro \\ California Institute of Technology
}

See next page for additional authors

Follow this and additional works at: https://pdxscholar.library.pdx.edu/phy_fac

Part of the Physics Commons

Let us know how access to this document benefits you.

Citation Details

Published as Nano Lett. 2020, 20, 11, 8127-8134. Publication Date:October 29, 2020. https://doi.org/ 10.1021/acs.nanolett.0c03159

This Post-Print is brought to you for free and open access. It has been accepted for inclusion in Physics Faculty Publications and Presentations by an authorized administrator of PDXScholar. Please contact us if we can make this document more accessible: pdxscholar@pdx.edu. 


\section{Authors}

Arash Farhadi, Manuel Bedrossian, Justin Lee, Gabrielle H. Ho, Mikhail G. Shapiro, and Jay Nadeau 


\title{
Genetically encoded phase contrast agents for digital holographic microscopy
}

\author{
Arash Farhadi, ${ }^{\dagger} \|$ Manuel Bedrossian, ${ }^{\ddagger}, \|$ Justin Lee, ${ }^{\dagger}$ Gabrielle H. Ho, " Mikhail \\ G. Shapiro, ${ }^{*}$ a and Jay L. Nadeau ${ }^{*, \S}$ \\ $\dagger$ Division of Biology and Biological Engineering, California Institute of Technology, \\ Pasadena, CA USA \\ $\ddagger$ Division of Engineering and Applied Science, California Institute of Technology, \\ Pasadena, CA USA \\ IDivision of Chemistry and Chemical Engineering, California Institute of Technology, \\ Pasadena, CA USA \\ $\S$ Department of Physics, Portland State University, Portland, OR USA \\ ||Equal Contribution \\ E-mail: mikhail@caltech.edu; nadeau@pdx.edu
}

\begin{abstract}
Quantitative phase imaging and digital holographic microscopy have shown great promise for visualizing the motion, structure and physiology of microorganisms and mammalian cells in three dimensions. However, these imaging techniques currently lack molecular contrast agents analogous to the fluorescent dyes and proteins that have revolutionized fluorescence microscopy. Here we introduce the first genetically encodable phase contrast agents based on gas vesicles. The relatively low index of refraction of the air-filled core of gas vesicles results in optical phase advancement relative to aqueous media, making them a "positive" phase contrast agent easily distinguished
\end{abstract}


from organelles, dyes, or microminerals. We demonstrate this capability by identifying and tracking the motion of gas vesicles and gas vesicle-expressing bacteria using digital holographic microscopy, and by imaging the uptake of engineered gas vesicles by mammalian cells. These results give phase imaging a biomolecular contrast agent, expanding the capabilities of this powerful technology for three-dimensional biological imaging.

\section{Keywords}

Quantitative Phase Imaging (QPI), Off-Axis Digital Holographic Microscopy, Volumetric Imaging, Phase Contrast Agent, Gas Vesicles, Reporter Genes, 3D Particle Tracking

\section{Introduction}

Precise acquisition of 4-dimensional data, comprising spatial coordinates and time, is important for studying many microscopic processes in biology. However, conventional optical microscopy suffers from a narrow depth of field due to its reliance on focusing lenses to encode spatial depth. Volumetric information is only obtained through step-wise acquisition of points or planes, and the resulting sequential acquisition typically necessitates low resolution in at least one of the 4 dimensions. Off-axis digital holographic microscopy (OADHM), an inherently volumetric recording technique, provides an alternative for instantaneous sampling of thick volumes. Successful applications have been made to studies of microbial motility, chemotaxis, flow of ions through ion channels, and migration of cancer cells ${ }^{1-7}$ including the use of 3-dimensional phase tomograms. ${ }^{8,9}$

With OADHM, optical interferometry is used to record a series of holograms at frame rates limited only by the camera. Off-line reconstruction yields plane-by-plane images of image intensity (brightfield) and phase. In OADHM, as in other forms of quantitative phase imaging $(\mathrm{QPI})$, phase contrast at any point $x, y$ is related to the difference in indices of 
refraction between the medium $\left(n_{m}\right)$ and objects in the light path $\left(n_{c}\right)$ multiplied by the object height $h:{ }^{10}$

$$
\Delta \phi=\frac{2 \pi}{\lambda} h(x, y)\left(n_{m}-n_{c}(x, y)\right)
$$

Because the typical values of $n_{c}$ for the cytoplasm and organelles are approximately $1.38,{ }^{11-13}$ which is very close to the $n_{m}$ of 1.33 for water, it is challenging for OADHM to visualize prokaryotic cells with much specificity, particularly when they are near the resolution limit of the instrument. ${ }^{14}$ This challenge could be overcome with suitable contrast agents or reporter genes, which would make cells more visible or highlight subcellular features and processes.

In fluorescence microscopy, this function is provided by targeted small-molecule dyes and fluorescent proteins, which have revolutionized the utility of this technique in biological research. ${ }^{15-18}$ Unfortunately, these same molecules are not effective as phase contrast agents. ${ }^{19-23}$ Although dyes create phase contrast related to absorbance by the KramersKronig relations, ${ }^{23}$ the necessary concentrations to create useful contrast cause cytotoxicity or, in the case of genetically encoded proteins, are impossible to achieve. Some designs for coupling OADHM with fluorescence imaging have been implemented, but they are complicated due to the incoherent nature of fluorescence emission and its weak signal. ${ }^{24-26}$

These issues can be addressed with genetically encodable phase contrast agents. An ideal phase contrast agent would have a more dramatically different index of refraction, and preferably one that is lower than that of $\mathrm{H}_{2} \mathrm{O}$ to be categorically distinguishable from other cellular components. Furthermore, an ideal contrast agent would demonstrate low toxicity to cells and be capable of sub-cellular targeting.

Here we introduce genetically encodable contrast agents for phase imaging. These contrast agents are based on a unique class of hollow protein nanostructures called gas vesicles $(\mathrm{GVs})$. GVs are all-protein nanostructures natively expressed in a number of waterborne microorganisms as a means to regulate their buoyancy. ${ }^{27,28} \mathrm{GVs}$ are air-filled compartments with dimensions on the order of $200 \mathrm{~nm}$, enclosed by a $2 \mathrm{~nm}$-thick protein shell (Figure 
1A), which is permeable to dissolved gas but prevents water from condensing into a liquid in the GV core. GVs have previously been described as contrast agents for ultrasound ${ }^{29}$ and magnetic resonance imaging, ${ }^{30-32}$ but have not been applied to optical microscopy. Recently, the genes encoding GVs have been heterologously expressed in commensal bacteria (e.g. Escherichia coli Nissle 1917 and Salmonella typhimurium) $)^{31,33}$ and mammalian cells. ${ }^{34}$

In this work, we test the hypothesis that the large difference in the index of refraction of GVs' gaseous interior $(n=1.0)$ relative to water and cytoplasm would produce strong positive phase contrast. We use a OADHM system capable of providing sub-micron resolution to visualize the phase contrast produced by purified GVs, allowing 4-dimensional tracking of their motion. In addition, we show that S. typhimurium cells expressing GVs as a genetically encoded reporter produce a unique pattern of phase contrast reflecting the sub-cellular distribution of these nanostructures. Furthermore, we demonstrate the use of GVs as targeted molecular contrast sources in mammalian cells by visualizing the uptake of engineered GVs into a mammalian cell line. This work establishes GVs as a tool for molecular and genetically encodable phase contrast, greatly expanding the utility of OADHM and related methods.

\section{Results and discussion}

\section{Gas vesicles produce positive phase contrast}

To establish the ability of GVs to produce phase contrast, we imaged GVs purified from $A n$ abaena flos-aquae. These nanostructures are approximately 120-140 nm wide and 200-800 nm long as measured by transmission electron microscopy (TEM) (Figure 1A). Individually, purified GVs were too small to resolve on our OADHM system, which uses numerical aperture (NA) of 0.3 aspheric lenses to achieve a spatial resolution of $0.8 \mu \mathrm{m}$ (Figure 1B). We therefore assembled GV clusters by biotinylation of the GVs followed by the addition of streptavidin. This yielded clusters with hydrodynamic diameters of $690 \pm 56 \mathrm{~nm}$ (Figure 1C 

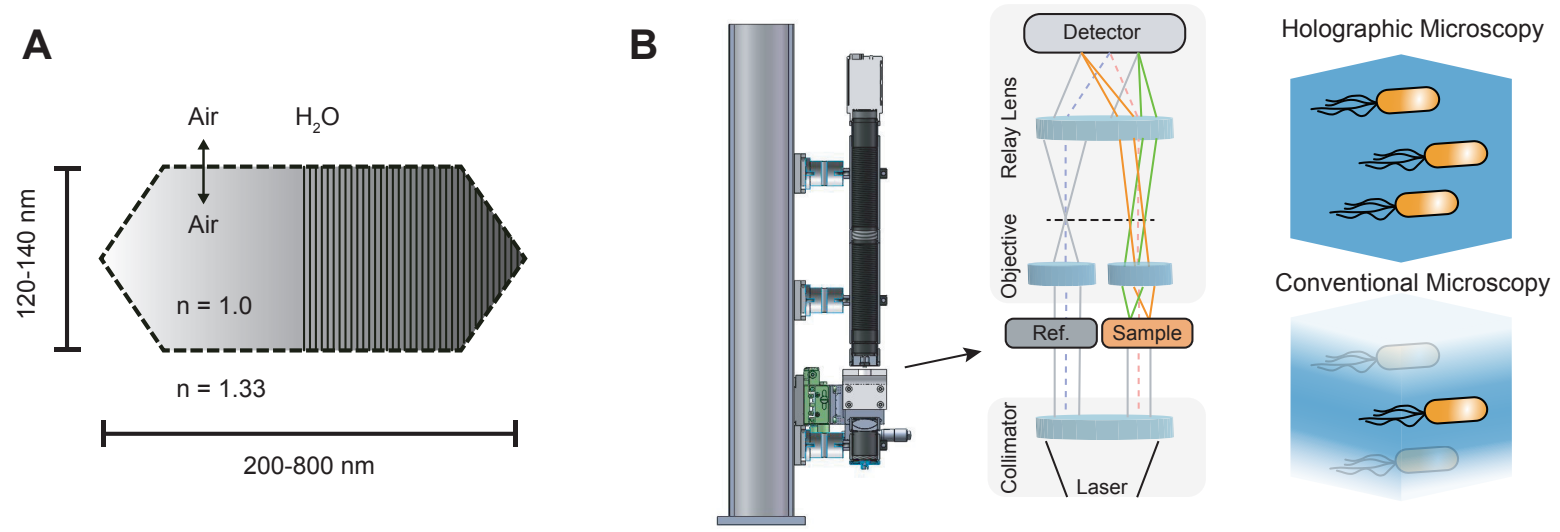

\section{C}

Streptavidin $>$
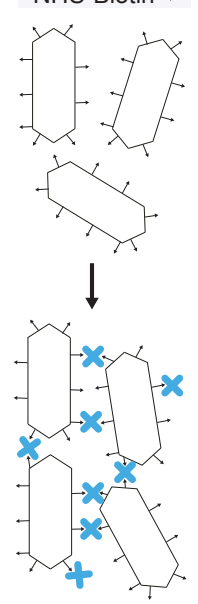

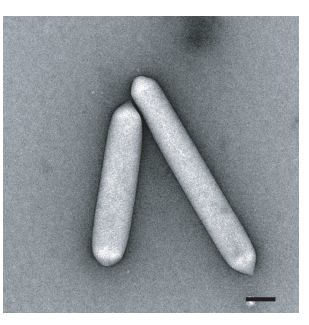

D

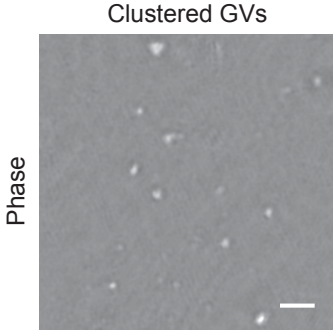

Alumina Beads
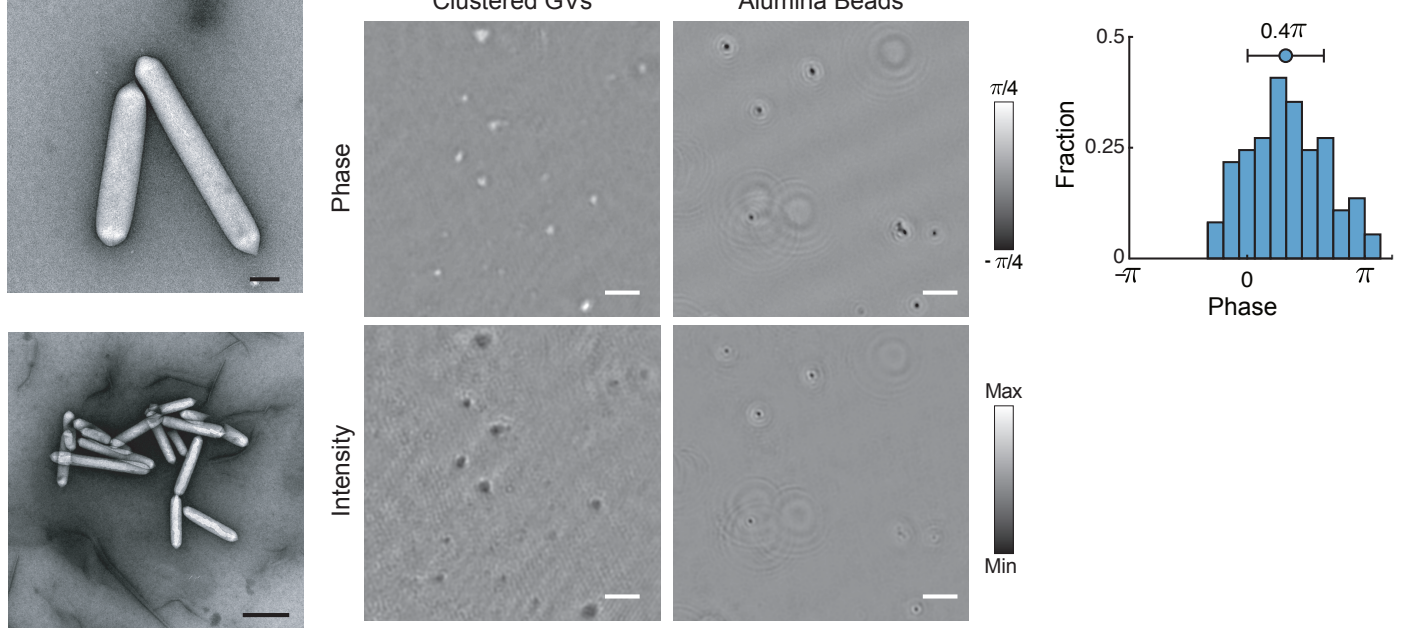

\section{E}

Figure 1: Gas vesicles as phase contrast agents. (A) Schematic of a single GV, indicating the index of refraction, $n$, inside the GV and in typical aqueous media. (B) Schematic of common path OADHM system used in this work, and an illustration of the inherent volumetric imaging of OADHM compared to conventional microscopy. (C) Biotinylated GVs purified from Anabaena flos-aquae can be clustered using streptavidin. Shown are a schematic and representative transmission electron micrograph of biotinylated GVs (top) and clustered GVs (bottom). Scale bars $100 \mathrm{~nm}$ and $500 \mathrm{~nm}$, respectively. (D) Representative OADHM phase and intensity images of clustered GVs and alumina beads. Scale bars represent 10 $\mu \mathrm{m}$. (E) Histogram of phase change observed from clustered GVs with apparent diameters from $0.6-2.2 \mu \mathrm{m}$. 
and Supplemental Figure 1). Holograms were collected of GV clusters and of comparably sized alumina beads $(1524 \pm 470 \mathrm{~nm}$ diameter as measured by dynamic light scattering) suspended in phosphate-buffered saline (PBS). We reconstructed these holograms into phase and intensity images as described in the supplementary text and Supplemental Figure 2. The phase shifts from the GV clusters and alumina beads were opposite in sign, with the GV clusters exhibiting positive phase contrast, while the alumina beads exhibited negative phase contrast (Figure 1D). Both particles appear dark in intensity images due to light scattering. The average phase shift produced by the GV clusters was $0.4 \pm 0.32 \pi$ (Figure 1E). GV phase contrast could be erased by irreversibly collapsing the GVs with hydrostatic pressure, ${ }^{35}$ providing a convenient internal control. After collapse, the positive phase contrast of GVs was eliminated (Supplemental Figure 3).

Having established GVs as phase contrast agents, we sought to determine if OADHM could be used to distinguish them and track their motion in a mixed particle population. ${ }^{36}$ First, we tracked the motion of both GV clusters and alumina beads in a $1 \mathrm{~mm}$-deep well. The average speed of GV clusters rising along the depth axis due to their buoyancy was $0.43 \pm 0.58 \mu \mathrm{m} \mathrm{s}^{-1}$ (95\% C.I.) (Figure 2A-C), while alumina beads sank with an average speed of $-0.47 \pm 1.02 \mu \mathrm{m} \mathrm{s}^{-1}$ (95\% C.I.) (Figure 2D-F). When GV clusters and alumina beads were mixed together and tracked, the two particle types were readily distinguished (Figure 2G-J), with the particles that were rising having positive phase contrast and the particles that were sinking having negative phase contrast (Figure 2K) with both particles appearing negative in intensity images (Supplemntary Figure 4). This demonstrated the ability of GVs to serve as a categorical contrast agent for 4-dimensional OADHM.

\section{Engineered gas vesicles serve as targeted contrast agents}

Among the advantages of $\mathrm{GVs}$ as phase contrast agents is their ability to be genetically engineered to modify their surface biochemical properties and enable molecular targeting. ${ }^{35,37}$ To demonstrate that engineered, targeted GVs could be used for phase imaging in living cells, 
A

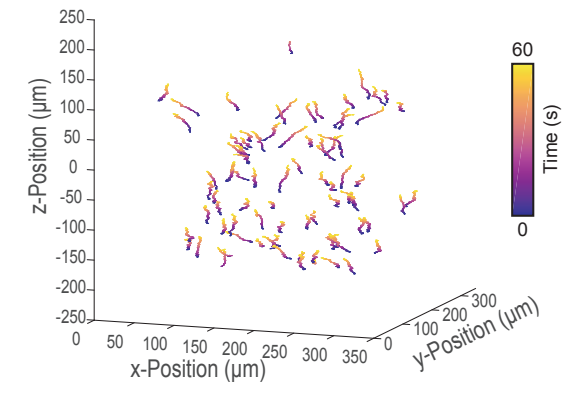

B

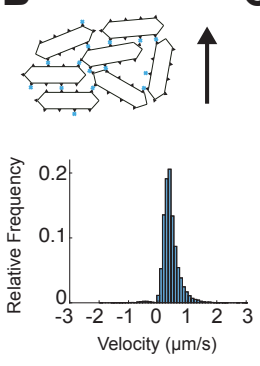

C

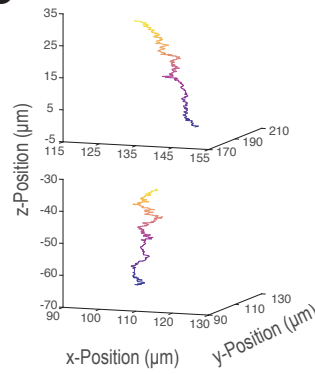

G

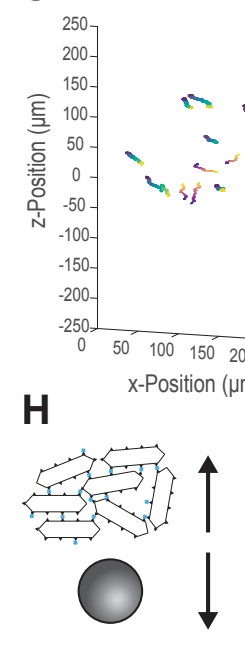

I
D

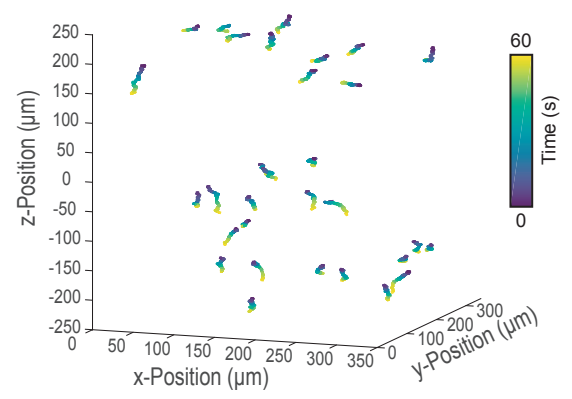

E

$F$
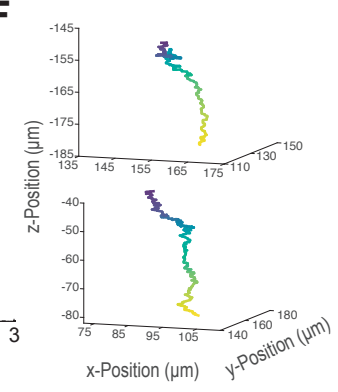

J

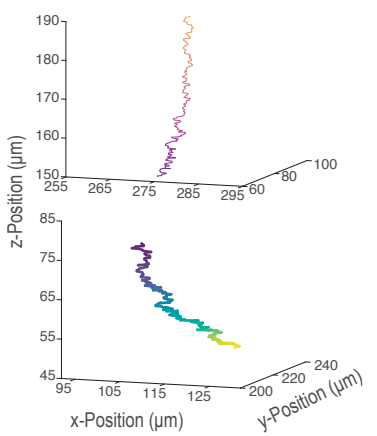

K

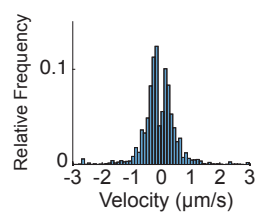

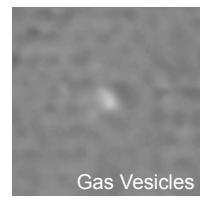

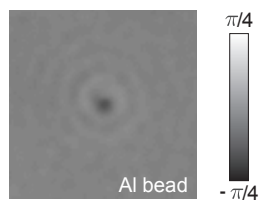

Figure 2: Volumetric tracking of particles in a mixed population suspension. (A) 3D trajectory plots of tracked GVs over 60 seconds. (B) Clustered GVs rise in solution (Top). Histogram of the z-component velocities of clustered GVs (Bottom). (C) Two example 3D tracks of clustered GVs. Each trajectory is color-coded with respect to time. (D) 3D trajectory plots of tracked alumina beads over 60 seconds. (E) Alumina beads sink over time (Top). Histogram of z-component velocities of alumina particles (Bottom). (F) Two example 3D tracks of alumina beads. Each trajectory is color-coded with respect to time. (G) 3D trajectory plots of the mixed population over 60 seconds. (H) Mixed population of clustered GVs and alumina beads. (I) Velocity histograms show two velocity populations, one for GVs and another for alumina beads. (J) Example trajectory of a rising and another of sinking particle chosen at random from G. (K) Phase images of the two particles from J. The buoyant particle has a positive phase contrast while the particle sinking has a negative phase contrast. Scale bars represent $5 \mu \mathrm{m}$. 
we used a fusion of the A. flos-aquae sequence to a polyarginine (R8) peptide. This peptide is a mimic of the human immunodeficiency virus 1 trans-activating (HIV-1 TAT) peptide and allows tagged proteins and particles to penetrate into mammalian cells (Figure 3A). ${ }^{38}$ Polyarginine-modified GVs were purified and added to Chinese hamster ovary (CHO) cells at $114 \mathrm{pM}$ for 45 minutes, washed and imaged by a OADHM system. This allowed for rapid 3-dimensional reconstruction of GV location on the surface of and within the cells (Figure 3B-D). GVs were fluorescently labeled with Alexa Fluor 488-NHS ester dyes to independently confirm the location of GV labeling on the cells (Figure 3E \& 3F). These experiments demonstrate the ability of GVs to label living cells for 3-dimensional phase imaging.

\section{Gas vesicles as genetically expressed contrast agents in Salmonella typhimurium}

Following the characterization of GVs as targeted OADHM contrast agents, we tested the ability of genetically encoded GVs to act as phase contrast reporter genes in living bacteria. A recently developed gene cluster encoding GVs, comprising a combination of genes from Anabaena flos-aquae and Bacillus megaterium, ${ }^{33}$ was used to heterologously express GVs in Salmonella typhimurium - a commonly used commensal bacteria for a number of cellular therapeutics applicaitons ${ }^{39}$ (Figure $\left.4 \mathrm{~A}\right)$. Upon induction with $\mathrm{N}$-( $\beta$-ketocaproyl)L-homoserine lactone (AHL), Salmonella formed numerous intracellular GVs, which were visualized under TEM and seen to typically cluster into distinct subcellular regions (Figure 4B). This pattern was expected to perturb the electromagnetic wavefront passing through a GV-expressing Salmonella cell so as to produce a distinct pattern in phase and intesnity images, as shown in simulated holograms (Figure 4C \& 4D, Supplemental Text).

As expected, under OADHM, GV-expressing Salmonella exhibited clear phase contrast that was different from control cells in which the GVs had been collapsed with hydrostatic pressure. ${ }^{35}$ Phase values of interior cellular regions of $50 \mathrm{GV}$-expressing salmonella cells 


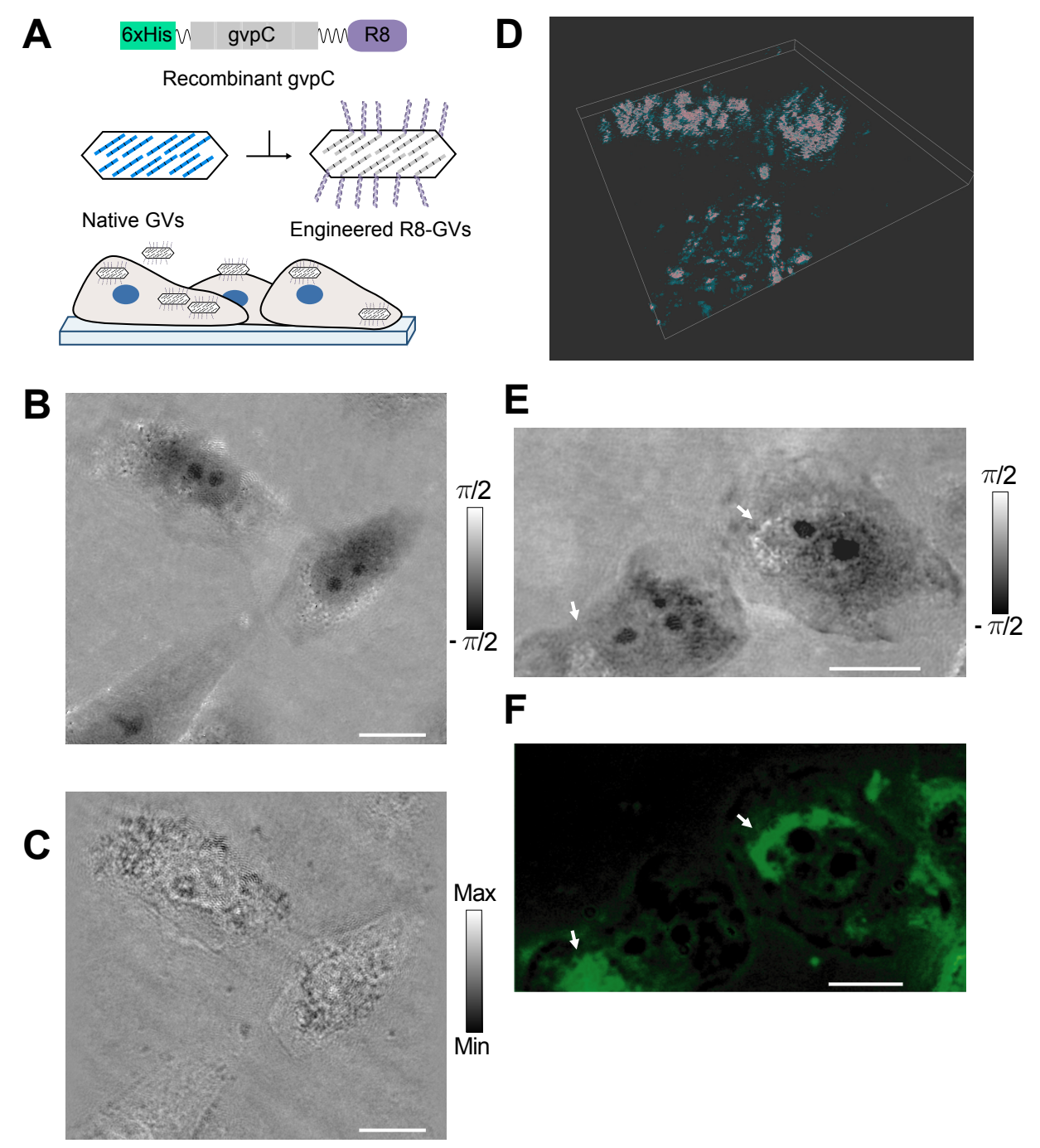

Figure 3: OADHM imaging of mammalian cells labeled with engineered gas vesicles. (A) Diagram of engineered GVs genetically functionalized with R8 peptides for attachment to and internalization by mammalian cells. Illustration of mammalian cells labeled with R8-GVs. (B) OADHM phase image of CHO cells labeled with R8-GVs. (c) Intesntiy image of CHO cells labeled with R8-GVs. Scale bar represents $25 \mu \mathrm{m}$. (D) Pseudocolored 3D rendering of GVs decorating CHO cells. For details see supplemental text. (E) Phase image of CHO cells labeled with Alexa Fluor 488 conjugated R8-GVs under the high power OADHM showing positive phase contrast in correspondence with $(\mathrm{F})$ a fluorescence image of the same $\mathrm{CHO}$ cells. Arrows indicate corresponding regions in E and F. All scale bars represent $25 \mu \mathrm{m}$. 
were analyzed and found to exhibit an average phase value of $0.06 \pm 0.05 \pi$ ( $95 \%$ C.I., $n=50$ cells) (Figure 4E \& 4G). The subcellular regions of these cells which contained high GV concentrations exhibited even higher phase values of $0.42 \pm 0.10 \pi$ ( $95 \%$ C.I., $n=110$ subcellular regions). The phase values of interior cellular regions of $50 \mathrm{GV}$-expressing Salmonella cells after their GVs were hydrostatically collapsed were measured to be $-0.06 \pm 0.10 \pi(95 \%$ C.I., $n=50$ cells) (Figure $4 \mathrm{~F} \& 4 \mathrm{G}$ ). The histogram of all pixels in Salmonella cells with and without GVs illustrates increased phase contrast due to GV-expression (Figure 4H). GV-expression increased the average signal to noise ratio of Salmonella cells in phase images by more than three-fold, making the cells easier to detect in solution.

Our results demonstrate that GVs serve as molecular and genetically encodable contrast agents for phase imaging due to the large difference in their index of refraction compared with aqueous media and organelles, resulting in positive phase contrast. The opposite sign of their refractive index difference from water compared to most other biological structures is especially beneficial for studying small samples such as microorganisms or subcellular features. The rapid volumetric image acquisition of OADHM makes it possible to identify and dynamically track GVs in a mixed solution containing other particles showing not only its ability to image a large volume at high resolution but its ability to maintain phase sensitivity across its depth of field. In addition, the modular protein make-up of GVs enables protein engineering to confer novel targeting properties for cellular imaging. This may facilitate future studies of cell-nanoparticle interactions and other dynamic cellular processes. Furthermore, by introducing GV gene encoded phase contrast, allowing cells activated to express these reporter genes to be distinguished from cells without reporter expression. These studies result in a QPI toolkit that will permit specific labeling in a large number of biological scenarios.

While the results presented in this study provide the key scientific evidence supporting the ability of GVs to serve as genetically encoded reporters for phase imaging, future work is needed to apply these agents to specific biological problems. To enable such applications, there exists significant scope for improvement and optimization. In using GVs as a contrast 


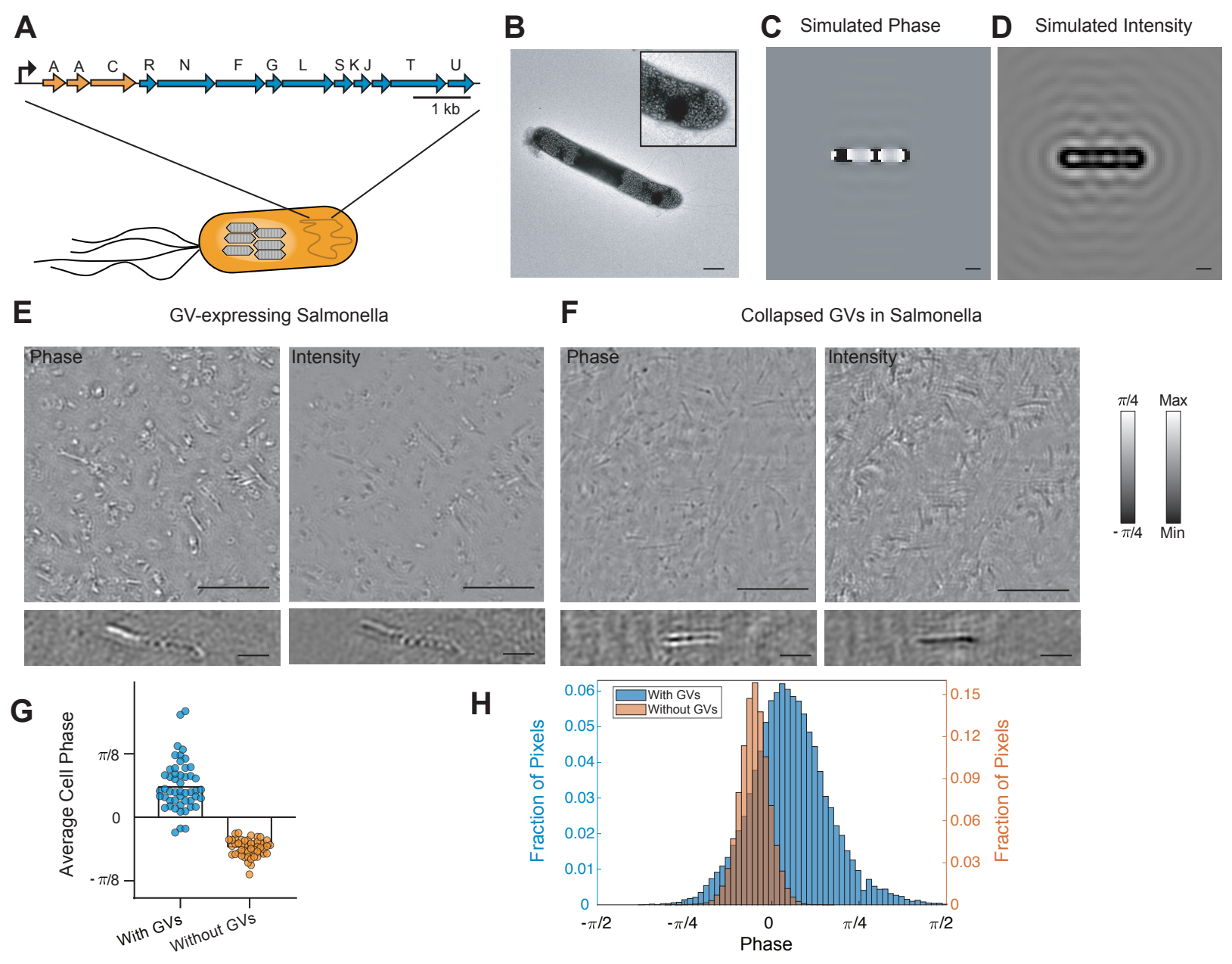

Figure 4: Gas vesicles as genetically encoded phase contrast agents in Salmonella. (A) Schematic of engineered GV gene cluster comprising genes from Anabaena flos-aquae (orange genes) and Bacillus megaterium (blue genes) that enable heterologous expression of GVs in Salmonella typhimurium. (B) Representative transmission electron micrograph of S. typhimurium expressing GVs. Inset is a $2 \mathrm{x}$ zoom in of a $\mathrm{GV}$ containing region of the salmonella cell. Scale bar represents $1 \mu \mathrm{m}$. (C) Numerical simulation of phase images of a GV-expressing Salmonella cell. (D) Numerical simulation of intensity images of a GVexpressing Salmonella cell. Scale bar represents $1 \mu \mathrm{m}$. (E) Representative phase (left) and intensity (right) images of GV-expressing S. typhimurium cells under OADHM. (F) Representative phase (left) and intensity (right) images of GV-expressing S. typhimurium cells after collapsing GVs using 1.2 $\mathrm{MPa}$ of hydrostatic pressure. Zoomed in images of a representative cell from $\mathrm{E}$ and $\mathrm{F}$ are shown on the bottom. Scale bars for full field of view are $25 \mu \mathrm{m}$, and $5 \mu \mathrm{m}$ for the zoomed in images. (G) Quantified average phase contrast from S. typhimurium cells with intact GVs compared with collapsed GVs ( $n=50$ cells). (H) Histogram of quantified phase contrast from all pixels in S. typhimurium cells with intact GVs $(n=50$ cells) compared with collapsed GVs $(n=50$ cells). 
agent for arbitrary samples, phase wrapping must be considered. In a typical QPI system, phase measurements are constrained to a modulo- $2 \pi$ (e.g. $-\pi$ to $\pi$ ). As a result, samples that introduce a phase shift of $\phi=2 \pi+m$, will only be seen to show a phase shift of $m$. Targeted efforts in the development of robust phase unwrapping algorithms will aid in the use of GVs in arbitrarily thick samples, eliminating the loss of contrast due to wrapping, ${ }^{40-45}$ Furthermore, robust phase unwrapping algorithms are needed to prevent the loss of positive phase contrast of GVs due to the thick negative phase contrast of typical intracellular material. Given the ability of GVs to be collapsed with acoustic pressure, additional contrast specificity could be obtained by integrating the ability to apply ultrasound waves to OADHM samples in situ and monitoring the resulting change in phase. ${ }^{31,33,35}$ In addition, the engineering of GV variants that collapse under different applied pressures may enable multiplexed phase contrast imaging. But it is expected that the greatest use of the technique will be in small, low-contrast cells and features, particularly for long-term recordings where phototoxicity of dyes and fluorescent proteins is a concern. Other immediate areas of application could be long-term, 4-dimensional tracking of bacterial motility and chemotaxis; engineering of subcellular- (e.g. mitochondrial) targeted GVs in order to explore changes in organelle shape and size with altered conditions; multimodal labeling to couple QPI with ultrasound and/or magnetic resonance imaging, particularly incorporating holographic tomography, for detection of labeled cancer cells or subcellular features. Furthermore, while genetic encoding facilitates the use of GVs to study genetically tractable organisms, there is also substantial interest in using OADHM for field studies, taking advantage of the microscopes' robust solid-state design. ${ }^{46}$ In such studies, the relevant microorganisms may be genetically intractable or their genetics entirely unknown, requiring the development of targeting moieties to bind GVs to such species. Such labeling would additionally facilitate the application of tracking and machine learning algorithms to the detection of small, near resolution limit, microorganisms in 4-dimensional data, where low image contrast is currently a challenge. ${ }^{47}$ As with all labeling techniques, possible cytotoxicity will need to be evaluated 
in the test system of interest. For studies of microbial behaviors such as motility and chemotaxis, it will be necessary to quantify the effect of genetically encoded and/or aptamer-bound GVs on these behaviors. We have seen elongation of Salmonella cells overexpressing GVs, indicating inhibition of cell division, and possible reduced motility. Extracellular labeling or reducing the expression levels may solve this problem. We anticipate that the development of dedicated molecular and genetically encodable contrast agents will usher in a new phase in holographic microscopy.

\section{Acknowledgement}

Arash Farhadi was supported by the NSERC graduate fellowship. Research in the Shapiro lab was supported by the National Institutes of Health (R01EB018975), the Packard Fellowship in Science and Engineering, the Pew Scholarship in the Biomedical Sciences and the Heritage Medical Research Institute. The Nadeau lab was supported by the Gordon and Betty Moore Foundation (Grant \#4038) and the National Science Foundation (NSF) 1828793. Portions of this work were supported under a contract from the Jet Propulsion Laboratory (JPL), California Institute of Technology, under a contract with the National Aeronautics and Space Administration (NASA). The authors thank Raymond Bourdeau for assistance with initial experiments, Kurt Liewer for the hologram simulation code and Dina Malounda for providing some of the samples.

\section{Supporting Information Available}

Experimental materials and methods. Raw data files as well as MATLAB code for the hologram simulations are included. Files:

S1: Raw holograms of GV clusters imaged with a coherent $405 \mathrm{~nm}$ laser (file type, TIFF)

S2: Raw holograms of alumina beads imaged with a coherent $405 \mathrm{~nm}$ laser (file type, TIFF) 
S3: Raw holograms of GV clusters imaged in solution with a coherent $405 \mathrm{~nm}$ laser (file type, TIFF)

S4: Raw holograms of alumina beads imaged in solution with a coherent $405 \mathrm{~nm}$ laser (file type, TIFF)

S5: Raw holograms of GV clusters and alumina beads imaged in solution with a coherent $405 \mathrm{~nm}$ laser (file type, TIFF)

S6: Raw holograms of $\mathrm{CHO}$ cells with GV imaged with a coherent $405 \mathrm{~nm}$ laser (file type, TIFF)

S7: Raw holograms of GV expressing salmonella cells imaged with a coherent $405 \mathrm{~nm}$ laser (file type, TIFF)

S8: Raw holograms of GV expressing salmonella cells after GVs were hydrostatically collapsed. Imaged with a coherent $405 \mathrm{~nm}$ laser (file type, TIFF)

S9: MATLAB code of hologram simulation suite (file type, M)

\section{References}

(1) Dubois, F.; Yourassowsky, C.; Monnom, O.; Legros, J.-C.; Debeir, O.; Van Ham, P.; Kiss, R.; Decaestecker, C. Digital holographic microscopy for the three-dimensional dynamic analysis of in vitro cancer cell migration. Journal of biomedical optics $\mathbf{2 0 0 6}$, 11, 054032 .

(2) Miniotis, M. F.; Mukwaya, A.; Wingren, A. G. Digital holographic microscopy for non-invasive monitoring of cell cycle arrest in L929 cells. PloS one 2014, 9, e106546.

(3) Jourdain, P.; Pavillon, N.; Moratal, C.; Boss, D.; Rappaz, B.; Depeursinge, C.; Marquet, P.; Magistretti, P. J. Determination of transmembrane water fluxes in neurons elicited by glutamate ionotropic receptors and by the cotransporters $\mathrm{KCC} 2$ and NKCC1: a digital holographic microscopy study. Journal of Neuroscience 2011, 31, 11846-11854. 
(4) Kemper, B.; Carl, D. D.; Schnekenburger, J.; Bredebusch, I.; Schäfer, M.; Domschke, W.; von Bally, G. Investigation of living pancreas tumor cells by digital holographic microscopy. Journal of biomedical optics 2006, 11, 034005.

(5) Pavillon, N.; Kühn, J.; Moratal, C.; Jourdain, P.; Depeursinge, C.; Magistretti, P. J.; Marquet, P. Early cell death detection with digital holographic microscopy. PloS one 2012, 7, e30912.

(6) Rappaz, B.; Breton, B.; Shaffer, E.; Turcatti, G. Digital holographic microscopy: a quantitative label-free microscopy technique for phenotypic screening. Combinatorial

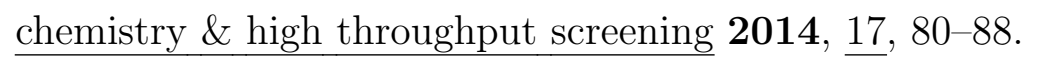

(7) Sheng, J.; Malkiel, E.; Katz, J.; Adolf, J.; Belas, R.; Place, A. R. Digital holographic microscopy reveals prey-induced changes in swimming behavior of predatory dinoflagellates. Proceedings of the National Academy of Sciences 2007, 104, 17512-17517.

(8) Merola, F.; Memmolo, P.; Miccio, L.; Savoia, R.; Mugnano, M.; Fontana, A.; D’ippolito, G.; Sardo, A.; Iolascon, A.; Gambale, A., et al. Tomographic flow cytometry by digital holography. Light: Science \& Applications 2017, 6, e16241-e16241.

(9) Miccio, L.; Cimmino, F.; Kurelac, I.; Villone, M. M.; Bianco, V.; Memmolo, P.; Merola, F.; Mugnano, M.; Capasso, M.; Iolascon, A., et al. Perspectives on liquid biopsy for label-free detection of "circulating tumor cells" through intelligent lab-onchips. View 2020, 20200034.

(10) Rappaz, B.; Marquet, P.; Cuche, E.; Emery, Y.; Depeursinge, C.; Magistretti, P. J. Measurement of the integral refractive index and dynamic cell morphometry of living cells with digital holographic microscopy. Optics express 2005, 13, 9361-9373.

(11) Bryant, F. D.; Seiber, B.; Latimer, P. Absolute optical cross sections of cells and chloroplasts. Archives of biochemistry and biophysics 1969, 135, 97-108. 
(12) Ross, K.; Billing, E. The water and solid content of living bacterial spores and vegetative cells as indicated by refractive index measurements. Microbiology 1957, 16, 418-425.

(13) Balaev, A. E.; Dvoretski, K. N.; Doubrovski, V. A. Determination of refractive index of rod-shaped bacteria from spectral extinction measurements. Saratov Fall Meeting 2002: Optical Technologies in Biophysics and Medicine IV. 2003; pp 375-380.

(14) Bedrossian, M.; Nadeau, J. L.; Serabyn, E.; Lindensmith, C. Sources and propagation of errors in quantitative phase imaging techniques using optical interferometry. Quantitative Phase Imaging III. 2017; p 100740E.

(15) Atanasova, M.; Yordanova, G.; Nenkova, R.; Ivanov, Y.; Godjevargova, T.; Dinev, D. Brewing yeast viability measured using a novel fluorescent dye and image cytometer. Biotechnology \& Biotechnological Equipment 2019, 33, 548-558.

(16) LV, W.; Gao, T.; Wang, S.; Hou, J.; Liu, M.; Yang, J.; Du, T.; Chen, Z.; Chen, Z.; Feng, X., et al. Long-Term Tracking of Cancer Cell Nucleus and Identification of Colorectal Cancer with an Aggregation-Induced Emission-Based Fluorescent Probe.

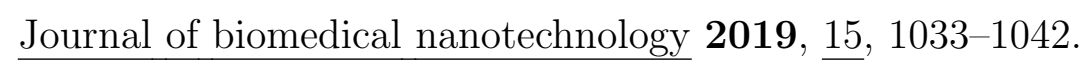

(17) Papadopoulos, J.; Müller, T. J. Rapid synthesis of 4-alkynyl coumarins and tunable electronic properties of emission solvatochromic fluorophores. Dyes and Pigments 2019, $\underline{166}, 357-366$.

(18) Xue, J.; Li, Z.; Xu, H.; Pu, Y. A novel fluorescent gold nanoparticle inhibiting migration and invasion of tumor cells. Reporters, Markers, Dyes, Nanoparticles, and Molecular Probes for Biomedical Applications XI. 2019; p 108930S.

(19) Gaigalas, A. K.; Choquette, S.; Zhang, Y.-Z. Measurement of scattering and absorption cross sections of dyed microspheres. Journal of research of the National Institute of

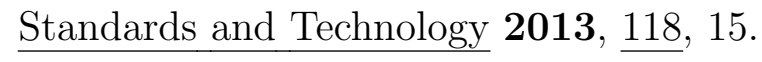


(20) Cherkezyan, L.; Subramanian, H.; Stoyneva, V.; Rogers, J. D.; Yang, S.; Damania, D.; Taflove, A.; Backman, V. Targeted alteration of real and imaginary refractive index of biological cells by histological staining. Optics letters 2012, 37, 1601-1603.

(21) Rappaz, B.; Charrière, F.; Depeursinge, C.; Magistretti, P. J.; Marquet, P. Simultaneous cell morphometry and refractive index measurement with dual-wavelength digital holographic microscopy and dye-enhanced dispersion of perfusion medium. Optics letters 2008, 33, 744-746.

(22) Nadeau, J. L.; Cho, Y. B.; Kühn, J.; Liewer, K. Improved tracking and resolution of bacteria in holographic microscopy using dye and fluorescent protein labeling. Frontiers in chemistry 2016, $\underline{4}, 17$.

(23) Nadeau, J. L.; Cho, Y. B.; Lindensmith, C. A. Use of dyes to increase phase contrast for biological holographic microscopy. Optics letters 2015, 40, 4114-4117.

(24) Mann, C. J.; Bingham, P. R.; Lin, H. K.; Paquit, V. C.; Gleason, S. S. Dual modality live cell imaging with multiple-wavelength digital holography and epi-fluorescence. 3D $\underline{\text { Research 2011, } 2,5 .}$

(25) Antonopoulos, C.; Kerasiotis, F.; Koulamas, C.; Papadopoulos, G.; Koubias, S. Experimental evaluation of the waspmote platform power consumption targeting ambient sensing. 2015 4th Mediterranean Conference on Embedded Computing (MECO). 2015; pp $124-128$.

(26) Nadeau, J. L.; Bedrossian, M.; Lindensmith, C. A. Imaging technologies and strategies for detection of extant extraterrestrial microorganisms. Advances in Physics: X 2018, $\underline{3}, 1424032$.

(27) Pfeifer, F. Distribution, formation and regulation of gas vesicles. Nature Reviews

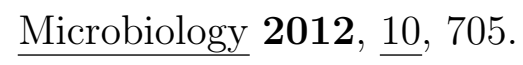


(28) Walsby, A. E. Gas vesicles. Microbiology and Molecular Biology Reviews 1994, 58, $94-144$.

(29) Shapiro, M. G.; Goodwill, P. W.; Neogy, A.; Yin, M.; Foster, F. S.; Schaffer, D. V.; Conolly, S. M. Biogenic gas nanostructures as ultrasonic molecular reporters. Nature nanotechnology 2014,, 9311.

(30) Shapiro, M. G.; Ramirez, R. M.; Sperling, L. J.; Sun, G.; Sun, J.; Pines, A.; Schaffer, D. V.; Bajaj, V. S. Genetically encoded reporters for hyperpolarized xenon magnetic resonance imaging. Nature chemistry 2014, $\underline{6}, 629$.

(31) Lu, G. J.; Farhadi, A.; Szablowski, J. O.; Lee-Gosselin, A.; Barnes, S. R.; Lakshmanan, A.; Bourdeau, R. W.; Shapiro, M. G. Acoustically modulated magnetic resonance imaging of gas-filled protein nanostructures. Nature materials 2018, 17, 456.

(32) Farhadi, A.; Ho, G.; Kunth, M.; Ling, B.; Lakshmanan, A.; Lu, G. J.; Bourdeau, R. W.; Schröder, L.; Shapiro, M. G. Recombinantly expressed gas vesicles as nanoscale contrast agents for ultrasound and hyperpolarized MRI. AIChE Journal 2018, 64, 2927-2933.

(33) Bourdeau, R. W.; Lee-Gosselin, A.; Lakshmanan, A.; Farhadi, A.; Kumar, S. R.; Nety, S. P.; Shapiro, M. G. Acoustic reporter genes for noninvasive imaging of microorganisms in mammalian hosts. Nature 2018, 553, 86.

(34) Farhadi, A.; Ho, G. H.; Sawyer, D. P.; Bourdeau, R. W.; Shapiro, M. G. Ultrasound imaging of gene expression in mammalian cells. Science 2019, 365, 1469-1475.

(35) Lakshmanan, A.; Farhadi, A.; Nety, S. P.; Lee-Gosselin, A.; Bourdeau, R. W.; Maresca, D.; Shapiro, M. G. Molecular engineering of acoustic protein nanostructures.

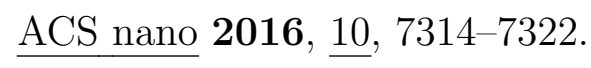

(36) Memmolo, P.; Miccio, L.; Paturzo, M.; Di Caprio, G.; Coppola, G.; Netti, P. A.; 
Ferraro, P. Recent advances in holographic 3D particle tracking. Advances in Optics and Photonics 2015, 7, 713-755.

(37) Lakshmanan, A.; Lu, G. J.; Farhadi, A.; Nety, S. P.; Kunth, M.; Lee-Gosselin, A.; Maresca, D.; Bourdeau, R. W.; Yin, M.; Yan, J., et al. Preparation of biogenic gas vesicle nanostructures for use as contrast agents for ultrasound and MRI. Nature protocols 2017, 12, 2050.

(38) Brock, R. The uptake of arginine-rich cell-penetrating peptides: putting the puzzle together. Bioconjugate chemistry 2014, 25, 863-868.

(39) Riglar, D. T.; Silver, P. A. Engineering bacteria for diagnostic and therapeutic applications. Nature Reviews Microbiology 2018, 16, 214.

(40) Ghiglia, D. C.; Romero, L. A. Robust two-dimensional weighted and unweighted phase unwrapping that uses fast transforms and iterative methods. JOSA A 1994, 11, 107117.

(41) Kaufmann, G. H.; Galizzi, G. E.; Ruiz, P. D. Evaluation of a preconditioned conjugategradient algorithm for weighted least-squares unwrapping of digital speckle-pattern interferometry phase maps. Applied optics 1998, 37, 3076-3084.

(42) Herráez, M. A.; Burton, D. R.; Lalor, M. J.; Gdeisat, M. A. Fast two-dimensional phase-unwrapping algorithm based on sorting by reliability following a noncontinuous path. Applied optics 2002, 41, 7437-7444.

(43) Bian, Y.; Mercer, B. Weighted regularized preconditioned conjugate gradient (PCG) phase unwrapping method. Journal of Optics A: Pure and Applied Optics 2008, $\underline{11}$, 015504 .

(44) Zuo, C.; Huang, L.; Zhang, M.; Chen, Q.; Asundi, A. Temporal phase unwrapping 
algorithms for fringe projection profilometry: A comparative review. Optics and Lasers in Engineering 2016, 85, 84-103.

(45) Yu, H.; Lan, Y.; Yuan, Z.; Xu, J.; Lee, H. Phase Unwrapping in InSAR: A Review. IEEE Geoscience and Remote Sensing Magazine 2019, 7, 40-58.

(46) Lindensmith, C. A.; Rider, S.; Bedrossian, M.; Wallace, J. K.; Serabyn, E.; Showalter, G. M.; Deming, J. W.; Nadeau, J. L. A submersible, off-axis holographic microscope for detection of microbial motility and morphology in aqueous and icy environments.

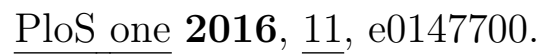

(47) Bedrossian, M.; El-Kholy, M.; Neamati, D.; Nadeau, J. L. A machine learning algorithm for identifying and tracking bacteria in three dimensions using Digital Holographic Microscopy. AIMS Biophysics 2018, 5, 36-49. 


\section{Graphical TOC Entry}

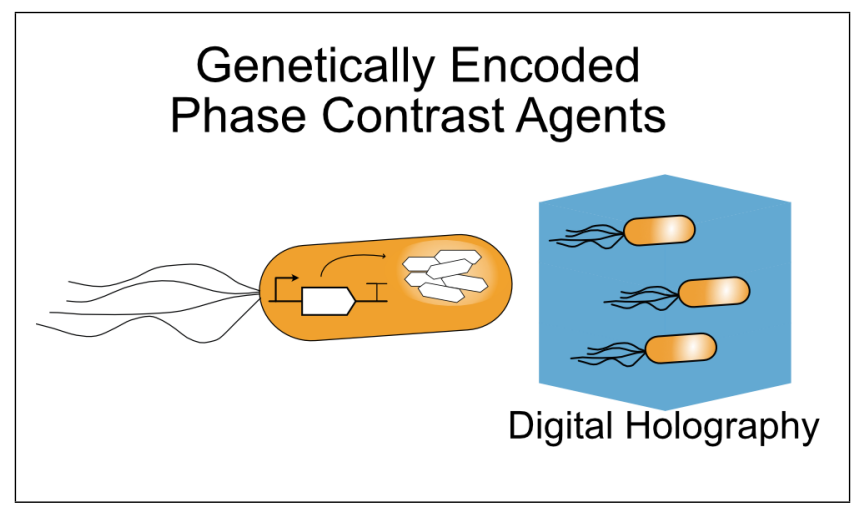

